\title{
Avaliação da qualidade de vida de cuidadores de afásicos
}

\section{Evaluation of the quality of life of caregivers of aphasic patients}

\author{
Ivone Panhoca ${ }^{1}$, Aline Nascimento Rodrigues ${ }^{2}$
}

\begin{abstract}
RESUMO
Objetivo: Avaliar a qualidade de vida de cuidadores de afásicos utilizando-se o The Medical Outcomes Study Short Form 36 (SF-36). Métodos: Foram analisados 30 cuidadores de pacientes afásicos, de ambos os gêneros, na faixa etária de 16 a 70 anos. Do grupo controle fizeram parte 30 sujeitos, totalizando assim 60 sujeitos. Todos foram convidados a responder às questões do SF-36. Além disso, foram consideradas as variáveis: gênero, idade, há quanto tempo ocupa a posição de cuidador, grau de parentesco com o paciente, e comprometimento motor (marcha) do afásico. Foram feitas análises de regressão univariada e descritivas para testar as associações entre as variáveis. As pontuações receberam tratamento estatístico utilizando-se o teste U de Mann-Whitney, considerados os objetivos propostos. Resultados: Variáveis como idade, tempo que exerce a função de cuidador, parentesco e comprometimento motor (marcha) da pessoa sob cuidados influenciaram significantemente as questões referentes aos aspectos físicos. Nos aspectos emocionais foram significativas apenas as variáveis idade e parentesco. Nos domínios referentes aos aspectos globais a única variável que influenciou no escore final foi o tempo de exercício da função de cuidador. Em relação aos aspectos físicos e globais, os cuidadores apresentaram escores estatisticamente iguais, enquanto que em relação aos aspectos emocionais observou-se que cuidadores tiveram menor escore do que o grupo controle. Conclusão: Em aspectos físicos e condições gerais, cuidadores e grupo controle não apresentaram diferenças consideráveis. Já em relação aos aspectos emocionais, os cuidadores apresentam-se mais comprometidos do que o grupo controle.
\end{abstract}

Descritores: Cuidadores; Assistência ao paciente; Afasia; Atenção à saúde; Qualidade de vida; Acidente vascular cerebral; Linguagem; Questionários

\section{INTRODUÇÃO}

A afasia se caracteriza por alterações dos processos lingüísticos de significação de origem articulatória e discursiva, produzidas por lesão focal adquirida no sistema nervoso central, em zonas responsáveis pela linguagem ${ }^{(1)}$. Tal prejuízo na linguagem pode causar ao indivíduo afásico uma restrição de comunicação com o meio, afetando as interações familiares e o convívio social ${ }^{(2)}$.

Além dos comprometimentos lingüísticos e cognitivos, com grande frequiência, os sujeitos afásicos apresentam comprometimentos motores, que os tornam ainda mais dependentes dos cuidadores, pois podem estar incapazes para exercer suas atividades de vida diária ${ }^{(3)}$.

Alguns autores referem que cuidadores familiares de pessoas com inabilidades tornou-se mais comum nos Estados

Trabalho realizado na Pontifícia Universidade Católica de Campinas PUCCamp - Campinas (SP), Brasil.

(1) Professora titular da Pontifícia Universidade Católica de Campinas PUCCamp - Campinas (SP), Brasil.

(2) Graduanda do Curso de Fonoaudiologia da Pontifícia Universidade Católica de Campinas - PUCCamp - Campinas (SP), Brasil.

Endereço para correspondência: Ivone Panhoca. Estrada da Rhodia, 5150,

Condomínio Residencial Colina, casa 28, Barão Geraldo, Campinas (SP),

Brasil, CEP: 13085-850. E-mail: i.panhoca@ terra.com.br

Recebido em: 26/8/2008; Aceito em: 21/11/2008
Unidos devido ao aumento na expectativa de vida e idade da população, resultando em alta prevalência de doenças crônicas e inabilidades associadas ${ }^{(4)}$. É importante se levar em consideração a desestruturação que se observa em familiares de pacientes adultos com acometimentos neurológicos crônicos ${ }^{(5)}$.

Cuidadores são indivíduos que têm a função de auxiliar e ou realizar a atenção adequada às pessoas que apresentam limitações para as atividades básicas da vida diária, estimulando a independência e respeitando a autonomia destas ${ }^{(6)}$.

$\mathrm{O}$ viver e conviver com pessoas que demandam cuidados, mesmo quando conduzido com acolhimento, afeto e ternura, é marcado por cansaço, estresse e esgotamento, o que coloca a família cuidadora na posição de necessitar, ela própria, de cuidado e de atenção ${ }^{(7)}$.

Em uma pesquisa com cuidadores familiares de pessoas que vivem em situação de doença crônica, concluiu-se que eles apresentaram alterações em sua qualidade de vida, sendo o bem-estar físico e psicológico significativamente afetados ${ }^{(8)}$.

Em estudo sobre cuidadores de pessoas pós AVC, os autores referem que tais pessoas mostraram comprometimentos emocionais significativos, o que ressalta a importância de um programa de assistência e orientação voltado para eles ${ }^{(9)}$.

Defende-se, no presente estudo, que, para que possa ser orientado, o cuidador precisa, antes, ser ouvido, ou seja: considerado, em toda sua dimensão social, cultural, econômica e afetivo-emocional. 
Dessa forma, essas pessoas não devem ser meramente "atendidas", mas sim acolhidas, e as intervenções a serem feitas junto a elas, no trabalho fonoaudiológico, devem ser construídas coletivamente, consideradas as demandas, as necessidades e as particularidades de cada sujeito e de cada família $^{(10)}$.

Especificamente em relação à linguagem, a afasia muitas vezes coloca o cuidador no papel de intérprete do sujeito cuja linguagem foi atingida pela lesão neurológica. Nesse contexto o cuidador passa a ser o mediador entre a pessoa sob seus cuidados e o mundo externo.

Sobre o poder das interações no processo de reconstrução da linguagem do paciente afásico a auto-confiança e autoestima têm a ver com o modo como os sujeitos se reorganizam como seres humanos que se comunicam. E os processos comunicativos se dão, sempre, em relação ao outro e nas relações com o outro ${ }^{(11-12)}$.

As experiências de cuidadores familiares devem receber atenção, com criação de novas políticas e de um sistema de prestação da formação e do apoio de que necessitam ${ }^{(13)}$. A orientação e a educação para a saúde podem gerar benefícios à recuperação do familiar sob cuidados e ainda proporcionar maior tranqüilidade e apoio aos familiares ${ }^{(3)}$.

O cuidador do paciente com seqüela de acidente vascular encefálico vive sob sobrecarga física e emocional constantes, uma vez que ao passar a ocupar a posição de cuidador ele passa a ter, além de sobrecarga de trabalho, responsabilidades, sobrecarga financeira e incertezas ${ }^{(3)}$.

O papel do cuidador é de extrema relevância na reintegração dos afásicos à sociedade, pois os sujeitos afásicos, pelas características motoras e lingüísticas que apresentam, são freqüentemente percebidos pelo grupo cultural como incapazes de usar as oportunidades sociais disponíveis, como as de trabalho ou de relações sociais, fazendo com que sejam discriminados ${ }^{(14)}$.

Especificamente as relações entre cuidadores e pessoas com história de acidentes vasculares cerebrais (não necessariamente afásicos) têm sido, no Brasil, objeto de estudos na área da enfermagem ${ }^{(7,15-17)}$.

$\mathrm{Na}$ área da saúde em geral, cuidadores de diferentes tipos de acometidos têm sido objeto de atenção e estudo na medicina, na fisioterapia e na terapia ocupacional o que pode ser observado tanto na literatura nacional ${ }^{(18-20)}$ quanto na internacional ${ }^{(9,13,21-24)}$.

Na Fonoaudiologia, no entanto, cuidadores ainda estão por ser devidamente estudados, sendo muito poucos os trabalhos desenvolvidos nessa área, em especial em relação aos cuidadores de sequelados neurológicos ${ }^{(2,25)}$.

O cuidador, nesse estudo, é a pessoa mais próxima do paciente; aquele que ou reside ou passa a maior parte do tempo com ele e participa ativamente do tratamento fonoaudiológi$\mathrm{Co}^{(20)}$. Portanto não estamos nos referindo, nesse trabalho, ao "cuidador profissional".

E ao falar em qualidade de vida estamos nos referindo, aqui, ao conceito da Organização Mundial de Saúde, que a define como "a percepção do indivíduo de sua posição na vida no contexto da cultura e sistema de valores nos quais ele vive e em relação aos seus objetivos, expectativas, padrões e preocupações" (26), uma vez que esse é o conceito mais adotado em estudos da área ${ }^{(27)}$.

Essa pesquisa teve como objetivo avaliar a qualidade de vida de cuidadores de afásicos, de ambos os gêneros, utilizando-se o The Medical Outcomes Study Short Form 36 (MOS-36 SF-36), instrumento validado e genérico.

\section{MÉTODOS}

\section{Dados dos sujeitos e da instituição}

Trinta cuidadores de pacientes afásicos, de ambos os gêneros, na faixa etária de 16 a 70 anos, que freqüentam o serviço de Fonoaudiologia da clínica-escola da Pontifícia Universidade Católica de Campinas (PUCCamp), acompanhando seus familiares sob cuidados, foram convidados a responder às questões do The Medical Outcomes Study Short Form 36 (MOS-36 SF-36), um instrumento de medidas genéricas para avaliar qualidade de vida, já validado no Brasil ${ }^{(28)}$, o qual tem sido utilizado com cuidadores em diversos estudos ${ }^{(9,18-20)}$.

Além desses, outros 30 sujeitos também foram convidados a responder ao questionário citado acima, compondo o grupo controle, formado por sujeitos de ambos os gêneros e que, como os afásicos, variavam da faixa de adultos jovens a idosos, totalizando assim 60 sujeitos participantes do presente estudo.

Os cuidadores tinham idade entre 16 e 70 anos com média de 52,8 anos, sendo que $80 \%$ dos participantes eram mulheres. A média de idade dos sujeitos do grupo controle foi 47,8 anos, variando entre 21 e 70 anos, sendo que $60 \%$ do total eram mulheres.

\section{Critérios de inclusão e exclusão}

Foram incluídos nesse estudo os sujeitos que, à época da coleta de dados, acompanhavam afásicos sob cuidados na clínica-escola do curso de Fonoaudiologia, a que as autoras são vinculadas, na condição de cuidadores, e que aceitaram responder às questões do instrumento aqui utilizado e a assinar o Termo de Consentimento Livre e Esclarecido.

Entende-se, aqui, por cuidador a pessoa mais próxima do paciente; aquele que ou reside ou passa a maior parte do tempo com ele e participa ativamente do tratamento fonoaudiológico, tendo o sujeito afásico sob sua responsabilidade diária direta, em todas as atividades do dia-a-dia (alimentação, higiene pessoal, comunicação, etc.).

O grupo controle, como já foi dito aqui, foi composto por sujeitos de ambos os gêneros, sem nenhuma queixa de comprometimento neurológico e que, como os cuidadores, variavam da faixa de adultos jovens a idosos. E todos também concordaram em assinar o termo de consentimento livre e esclarecido.

\section{Coleta de dados}

O SF-36 é um questionário multidimensional, composto por 36 itens, dentro de dois grandes grupos: físico e mental. Cada um destes é formado por quatro domínios, que por sua vez, se constituem de itens que avaliam uma mesma área da vida do sujeito avaliado.

O sub-grupo físico é composto pelos domínios: capacidade 
funcional (10 itens); limitação por aspectos físicos (quatro itens); dor (dois itens); estado geral de saúde (cinco itens).

O sub-grupo mental abrange domínios como: vitalidade (quatro itens); aspectos sociais (dois itens); limitação por aspectos emocionais (três itens); saúde mental (cinco itens).

Há, ainda, uma questão de avaliação comparativa entre as condições atuais de saúde e as de um ano atrás.

Pode-se obter um escore final de cada domínio, que varia de 0 a 100, sendo que, quanto maior a pontuação, melhor é o estado de saúde do avaliado. Tal escala de cálculo é chamada de Raw Scale, pois o valor final não apresenta nenhuma unidade de medida, obtendo-se números simbólicos que definem o grau de comprometimento que o sujeito apresenta em cada domínio.

Além dos dados obtidos sob forma de respostas às questões apresentadas, foram consideradas as variáveis: gênero; idade; há quanto tempo ocupa a posição de cuidador, tipo de afásico sob cuidado (fluente ou não fluente e marcha independente ou dependente), além de grau de parentesco com o paciente; dados de extrema relevância ${ }^{(29)}$. Para a composição do grupo controle foram considerados gênero e idade.

Foi elaborada uma planilha em um programa digital, da qual consta uma listagem com todos os sujeitos da pesquisa cada qual com um código numérico adotado. Foram inseridos em uma segunda tabela todos os dados coletados no início da entrevista (variáveis citadas anteriormente).

Em uma terceira tabela, foram colocadas as respostas obtidas na aplicação do questionário e foi criada uma quarta planilha, na qual as respostas são resgatadas da anterior e as pontuações definidas são aplicadas para cada questão. Nessa mesma planilha as questões foram separadas em seus respectivos domínios (capacidade funcional, limitação por aspectos físicos, dor, estado geral de saúde, vitalidade, aspectos sociais, limitação por aspectos emocionais e saúde mental), e receberam o cálculo do Raw Scale, resultando em notas que variam de zero a 100, onde zero é o pior e 100 é o melhor para cada domínio.

\section{Análise dos dados}

Considerados os oito domínios oriundos do questionário SF-36, para reduzir o número de comparações e simplificar as análises estatísticas (e/ou interpretações), foram construídos fatores através da técnica de Análise Fatorial, utilizando o método de componentes principais com rotação ortogonal Varimax para estimação. Essas novas variáveis, não correlacionadas, foram construídas de tal forma que sumarizem as informações principais das variáveis originais.

Obtivemos, portanto os seguintes índices: componente para aspectos físicos (CAF), índice influenciado por valores de capacidade funcional, limitações de aspectos físicos e dor; qualidade de vida em termos globais (QVG), índice influenciado por valores de estado geral de saúde, vitalidade e saúde mental; componente para aspectos emocionais (CAE), índice influenciado por valores de aspectos sociais e limitação de aspectos emocionais. Esses fatores explicam $71,8 \%$ da variabilidade original dos dados.

Foi feita uma análise exploratória dos dados, para descrever algumas características, tais como média, mediana, desvio padrão, proporções, entre outras. Tais medidas foram utilizadas para formular hipóteses de interesse.

Para obter uma medida que descreve o relacionamento entre duas variáveis utilizou-se o coeficiente de correlação, o qual mostra se há um relacionamento linear entre duas variáveis. O coeficiente de correlação é uma medida que está entre -1 e 1. Valores próximos de 1 indicam uma forte associação linear positiva entre as variáveis e valores próximos de -1 indicam uma forte associação linear negativa entre as variáveis. Quando não há relacionamento entre duas variáveis, o coeficiente de correlação tende a estar próximo de zero.

Em análises estatísticas descritivas os escores dos grupos cuidadores e controle foram divididos por faixas etárias e gênero. Verificou-se se algumas das diferenças encontradas na análise descritiva são estatisticamente significativas ou não, através de testes de hipóteses. Esses testes foram avaliados através do p-valor de um teste, o qual é uma probabilidade que mensura o quão verossímil é nossa hipótese base. Quanto maior for o p-valor mais evidências a favor da hipótese nula temos. Rejeitou-se a hipótese nula para o p-valor menor que 5\% (com atenção para aqueles menores que 10\%).

Os testes descritos acima são testes não-paramétricos. Foi utilizado para todos os domínios e fatores, o teste de MannWhitney, verificando-se se haviam diferenças significativas entre os escores de cuidadores e escores de indivíduos do grupo controle.

Para avaliar a qualidade de vida dos cuidadores levando em consideração seus aspectos demográficos, foi utilizada a abordagem de regressão univariada, baseada nos índices CAF, QVG e CAE.

\section{Comitê de ética}

Os 60 sujeitos que fizeram parte desse estudo assinaram o Termo de Consentimento Livre e Esclarecido após o projeto ter sido aprovado pelo Comitê de Ética em Pesquisa da Pontifícia Universidade Católica de Campinas (PUCCamp), sob protocolo $\mathrm{n}^{\circ}$ 582/06.

\section{RESULTADOS}

Através da análise exploratória dos dados observou-se que nos domínios referentes aos aspectos físicos os cuidadores $(M=59,2)$ apresentaram escores maiores do que o grupo controle $(M=53,89)$. Uma relação inversa ocorre nos domínios referentes aos aspectos emocionais, sendo que o grupo controle $(M=62,27)$ apresentou maior escore do que os cuidadores $(\mathrm{M}=52,57)$.

Já nos domínios referentes aos aspectos mais gerais, foram obtidos valores bem próximos para os dois grupos como mostrado na Figura 1 (lembrando que os escores finais variam de zero a 100, sendo que, quanto maior a pontuação, melhor é o estado de saúde do avaliado).

Ao verificar o relacionamento linear entre algumas variáveis, utilizando o coeficiente de correlação de Spearman, observou-se uma relação significativa, considerando valores maiores que 0,6 , entre as seguintes variáveis para cuidadores 
Tais dados indicam que quanto maior o escore em uma dessas variáveis, maior o escore da outra.

Outro fato observado foi que os valores de correlação para o grupo de cuidadores é relativamente maior que os valores

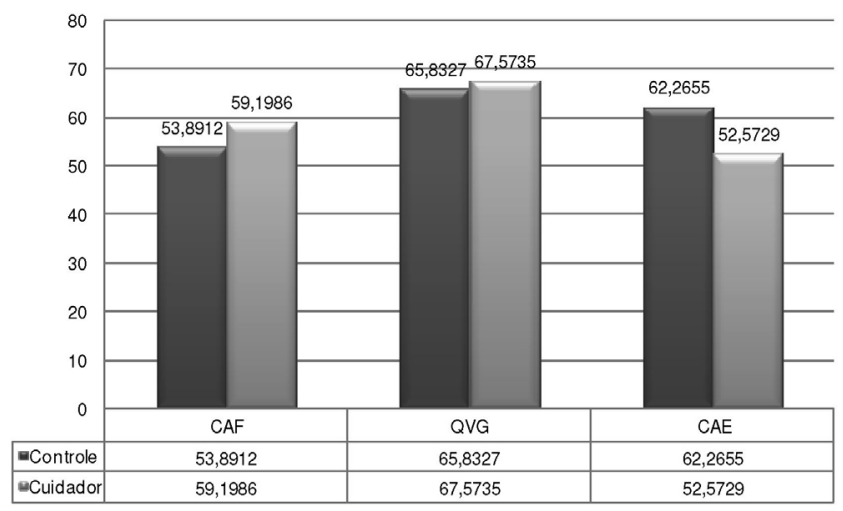

Figura 1. Comparação dos escores dos índices de resumo Legenda: $\mathrm{CAF}=$ componente para aspectos físicos; $\mathrm{QVG}$ = qualidade de vida em termos globais; $\mathrm{CAE}=$ componente para aspectos emocionais

Tabela 1. Coeficientes de correlação entre os domínios do grupo Cuidadores (Correlação de Spearman)

\begin{tabular}{lccccccc}
\hline & CF & LAF & D & EGS & V & AS & LAE \\
\hline LAF & 0,477 & & & & & & \\
D & $0,607^{*}$ & $0,677^{*}$ & & & & & \\
EGS & 0,527 & 0,44 & $0,605^{*}$ & & & & \\
V & 0,57 & 0,209 & 0,41 & 0,509 & & & \\
AS & 0,513 & 0,357 & 0,35 & 0,43 & 0,475 & & \\
LAE & 0,344 & 0,317 & 0,157 & 0,356 & 0,17 & 0,55 & \\
SM & 0,481 & 0,399 & 0,279 & 0,386 & 0,471 & $0,697^{*}$ & $0,737^{*}$ \\
\hline
\end{tabular}

Legenda: $\mathrm{CF}=$ capacidade funcional; $\mathrm{LAF}=$ limitação por aspectos físicos; $\mathrm{D}=$ dor; $\mathrm{EGS}$ = estado geral de saúde; $\mathrm{V}$ = vitalidade; $\mathrm{AS}$ = aspectos sociais; LAE = limitação por aspectos emocionais; $S M=$ saúde mental; * Valores acima de 0,6 (considerados relevantes na correlação entre os domínios apresentados) para o grupo controle, indicando assim um relacionamento mais acentuado entre os domínios do grupo cuidadores do que nos domínios do grupo controle.

Em análises estatísticas descritivas foram obtidas medidas de acordo com a idade e gênero de ambos os grupos. Para cuidadores, em relação à idade, nos domínios referentes aos aspectos físicos (CAF) o escore é menor em sujeitos com idade abaixo de 40 anos $(M=59,7)$ e com idade acima de 60 anos $(\mathrm{M}=51,9)$, assim como nos domínios referentes aos aspectos globais. Já nos domínios referentes aos aspectos emocionais (CAE) o escore é menor em sujeitos com idade abaixo de 40 anos $(M=43,4)$ e com idade entre 41-54 anos $(M=41,3)$, de acordo com a Tabela 2.

Para o grupo controle, no que diz respeito à idade, nos domínios referentes aos aspectos físicos (CAF) o escore é menor em sujeitos com idade entre 41-54 anos $(M=40,9)$ e com idade acima de 60 anos $(M=34,6)$; o mesmo se dá nos domínios referentes aos aspectos globais. Já nos domínios referentes aos aspectos emocionais (CAE) o escore é menor em sujeitos com idade entre 55-60 anos $(M=53,23)$ e com idade acima de 60 anos $(M=56,5)$.

Em relação ao gênero, analisando-se aspectos físicos, emocionais e globais os cuidadores do sexo masculino obtiveram escores maiores do que os do sexo feminino, como pode ser verificado na Tabela 3. Já no grupo controle, em aspectos físicos as mulheres obtiveram escores maiores $(\mathrm{M}=57,9)$ do que os homens $(\mathrm{M}=47,8)$.

Utilizando testes de hipóteses procurou-se averiguar em quais domínios (ou fatores) havia diferenças nos escores do grupo cuidadores e controle, buscando uma possível evidência de diferentes qualidades de vidas entre os dois grupos.

Estatisticamente não foram encontradas diferenças nos escores das duas populações, como pode ser observado na Tabela 4, o que mostra que a qualidade de vida dos cuidadores é equivalente à qualidade de vida do grupo controle. Baseados nos índices adquiridos a partir da análise fatorial, também não foram constatadas diferenças significativas entre os seus

Tabela 2. Escores obtidos através da análise das respostas do SF-36 do Grupo de Cuidadores de acordo com a variável faixa etária

\begin{tabular}{|c|c|c|c|c|c|c|c|c|}
\hline \multirow[t]{2}{*}{ Variável } & \multicolumn{2}{|c|}{$<40$} & \multicolumn{2}{|c|}{$41-54$} & \multicolumn{2}{|c|}{$55-60$} & \multicolumn{2}{|c|}{$>60$} \\
\hline & Média & DP & Média & DP & Média & DP & Média & DP \\
\hline CF & 84,0 & 17,4 & 84,1 & 12,8 & 86,6 & 12,5 & 78,5 & 21,4 \\
\hline LAF & 70,0 & 32,6 & 79,2 & 40,1 & 80,5 & 27,3 & 62,5 & 42,9 \\
\hline$D$ & 55,0 & 33,9 & 61,0 & 25,8 & 63,5 & 28,1 & 55,1 & 26,4 \\
\hline EGS & 64,2 & 29,4 & 86,3 & 13,0 & 76,3 & 17,6 & 75,4 & 17,7 \\
\hline V & 67,0 & 17,1 & 70,0 & 20,7 & 68,8 & 20,5 & 58,0 & 18,4 \\
\hline AS & 65,0 & 13,6 & 64,6 & 30,0 & 73,6 & 35,0 & 71,2 & 22,0 \\
\hline LAE & 33,3 & 47,1 & 50,0 & 46,0 & 66,7 & 40,8 & 76,7 & 41,7 \\
\hline SM & 55,2 & 12,4 & 62,0 & 17,3 & 67,1 & 16,8 & 63,6 & 26,5 \\
\hline $\mathrm{CAF}$ & 59,7 & 28,8 & 64,9 & 20,6 & 63,1 & 27,5 & 51,9 & 25,6 \\
\hline QGV & 61,4 & 19,8 & 74,7 & 13,1 & 69,7 & 15,8 & 64,4 & 23,7 \\
\hline CAE & 43,4 & 13,3 & 41,3 & 30,6 & 56,8 & 26,6 & 60,0 & 21,4 \\
\hline
\end{tabular}

Legenda: $\mathrm{DP}=$ desvio padrão; $C F=$ capacidade funcional; LAF = limitação por aspectos físicos; $\mathrm{D}=$ dor; $\mathrm{EGS}=$ estado geral de saúde; $\mathrm{V}=$ vitalidade; $\mathrm{AS}=$ aspectos sociais; LAE = limitação por aspectos emocionais; SM = saúde mental; CAF = componente para aspectos físicos; QGV = qualidade de vida em termos globais; $\mathrm{CAE}=$ componente para aspectos emocionais 
Tabela 3. Escores obtidos através da análise das respostas do SF-36 do grupo cuidadores versus grupo controle de acordo com a variável sexo

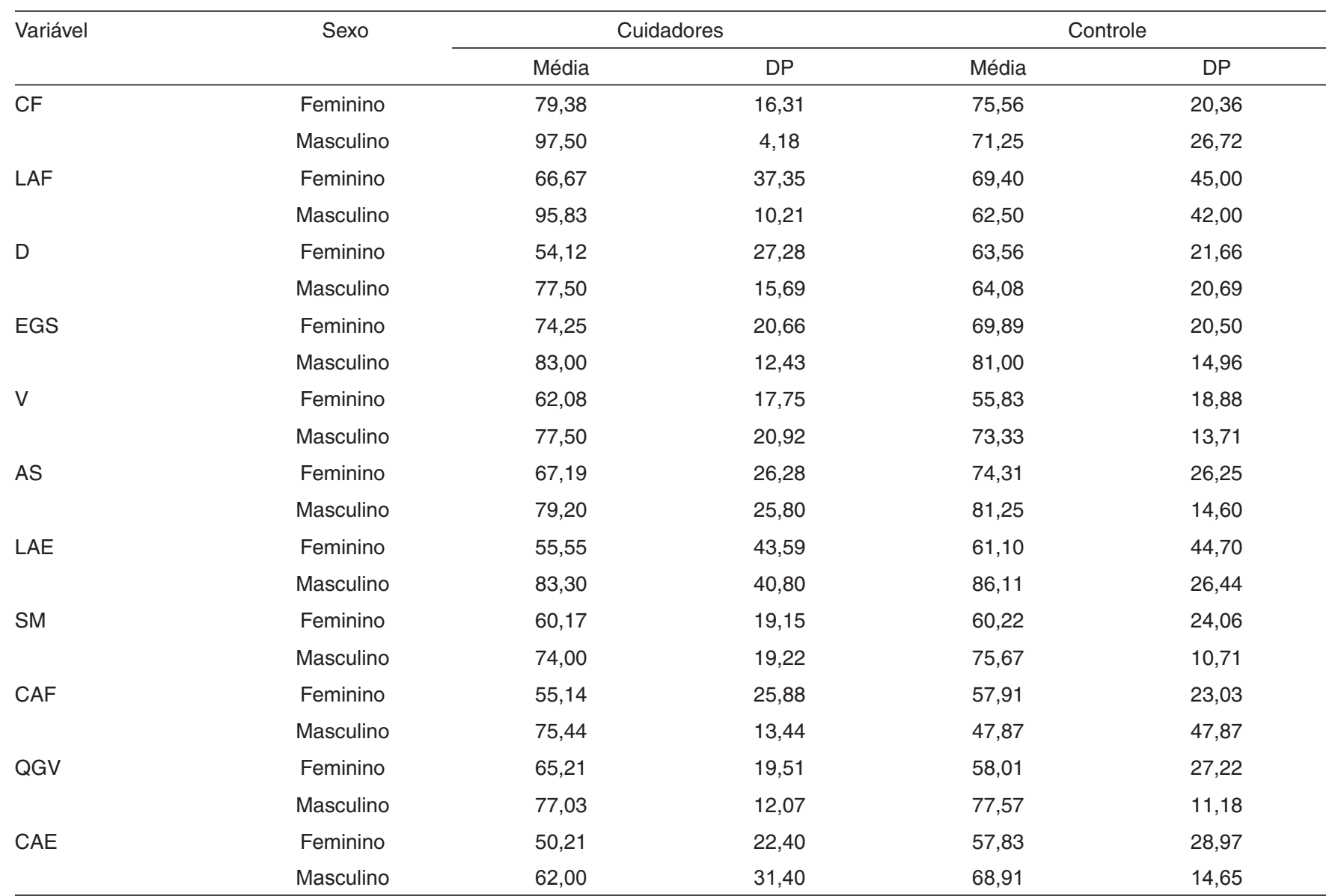

Legenda: DP = desvio padrão; CF = capacidade funcional; LAF = limitação por aspectos físicos; $D=$ dor; EGS = estado geral de saúde; V = vitalidade; $A S=$ aspectos sociais; LAE = limitação por aspectos emocionais; $\mathrm{SM}$ = saúde mental; CAF = componente para aspectos físicos; $\mathrm{QGV}=$ qualidade de vida em termos globais; $\mathrm{CAE}=$ componente para aspectos emocionais

Tabela 4. Escores obtidos através da análise das respostas do SF-36 do grupo cuidadores versus grupo controle

\begin{tabular}{lccc}
\hline Domínio & Controle & Cuidadores & Valor de $p$ \\
\hline CF & 82,5 & 87,5 & 0,13 \\
LAF & 100 & 100 & 0,87 \\
D & 62 & 67 & 0,66 \\
EGS & 77 & 78 & 0,61 \\
V & 65 & 62 & 0,67 \\
AS & 87,5 & 68,75 & 0,28 \\
LAE & 100 & 83,35 & 0,41 \\
SM & 72 & 66 & 0,35 \\
CAF & 54,46 & 67,13 & 0,35 \\
QGV & 71,58 & 59,65 & 0,98 \\
CAE & 65,04 & 56,81 & 0,11 \\
\hline
\end{tabular}

Testes de Mann-Whitney

Legenda: $C F$ = capacidade funcional; LAF = limitação por aspectos físicos; $\mathrm{D}=$ dor; $\mathrm{EGS}$ = estado geral de saúde $\mathrm{V}$ = vitalidade; $\mathrm{AS}$ = aspectos sociais; $\mathrm{LAE}$ = limitação por aspectos emocionais; $\mathrm{SM}$ = saúde mental; CAF = componente para aspectos físicos; $\mathrm{QGV}=$ qualidade de vida em termos globais; CAE = componente para aspectos emocionais

escores para o grupo controle e para o grupo de cuidadores (Figura 1).

Foram também realizados os mesmos testes em outras variáveis, estratificados pelas variáveis demográficas. Com isso, procurou-se verificar se a qualidade de vida entre os grupos sofrem alteração quando são consideradas as variáveis demográficas idade e gênero.

Verificou-se que para os entrevistados do gênero masculino a qualidade de vida dos cuidadores, em aspectos físicos, está mais comprometida (CAF: $\mathrm{p}=0,1$ ) do que os do mesmo gênero do grupo controle.

Visando verificar como as variáveis demográficas influenciam da qualidade de vida dos cuidadores, utilizou-se a abordagem de regressão univariada, para os fatores criados na análise fatorial, na qual se observou que nos domínios referentes aos aspectos físicos (CAF) influenciaram significativamente a idade dos cuidadores, tempo na função de cuidador, parentesco e comprometimento motor (marcha) da pessoa sob cuidados.

Foi constatado que, para domínios referentes aos aspectos físicos (CAF), os cuidadores com idade mais elevada tiveram menor escore, sendo que para cada ano aumentado na idade espera-se uma diminuição no índice CAF em 0,74 pontos. No que se refere ao tempo de cuidado, os dados mostraram que há quanto mais tempo o cuidador exerce a função, menor é o escore de CAF, sendo esperada uma diminuição de 2,30 pontos nesse índice a cada ano a mais de cuidado. Já em relação ao parentesco foi adotada uma escala de 1 a 4 , sendo: 1 - mãe e pai; 2 - marido e esposa; 3 - filho; 4 - irmão. 
O escore da variável CAF para irmãos, filhos e maridos/ esposas pode ser considerado equivalente. Entretanto para pais/mães o escore tem aumento de 46 pontos em relação aos demais, indicando que em aspectos físicos as pessoas dessa categoria estão com melhor qualidade de vida.

Além disso, notou-se que quando o paciente é cadeirante, é esperado que o escore do cuidador para CAF diminua em aproximadamente 14 pontos, indicando assim uma piora em aspectos físicos para aqueles que cuidam de pacientes cadeirantes.

Nos domínios referentes aos aspectos emocionais (CAE), influenciaram significativamente a idade e o parentesco. Quanto à idade, os cuidadores de idade mais elevada tiveram menor escore no índice CAE, sendo que para cada ano aumentado espera-se que esse índice diminua 0,60 pontos. Utilizando a mesma escala descrita acima em relação ao grau de parentesco, foi observado que no grau 1 (mãe e pai) foram encontrados menores escores em CAE, enquanto que no grau 4 (irmão) foram encontrados os maiores escores, sendo que de um grau de parentesco a outro observa-se um aumento esperado de 10 pontos no escore.

Nos domínios referentes aos aspectos globais (QVG) influenciou significativamente apenas o tempo na função de cuidador, tendo-se observado que quanto maior esse tempo, menor é o escore de QGV, sendo que a cada ano a mais na função esse índice tem uma diminuição de 1,35.

\section{DISCUSSÃO}

Dentre os cuidadores aqui pesquisados houve a prevalência de mulheres $(80 \%)$ o que está em conformidade com numerosos estudos já realizados com cuidadores, não somente de pessoas com seqüela de acidente vascular cerebral ${ }^{(6,8-9,17,19,24)}$. Alguns autores destacam que há obrigação e dever embutidos ou no compromisso do matrimônio ou no traço cultural fortemente presente em nossa sociedade, que determinam que a mulher deve ocupar o papel de cuidadora, seja ela a esposa, a filha ou a neta do idoso ${ }^{(6,17)}$. Tal dado indica, portanto, que esse grupo merece atenção especial dos profissionais da área da saúde, dentre eles os fonoaudiólogos.

Nesse estudo foi realizada uma análise fatorial a partir dos dados coletados quanto ao questionário SF- 36 e foram obtidos três fatores que explicaram $71,8 \%$ da variabilidade original dos dados. Tal análise também foi realizada em estudos pré$\operatorname{vios}^{(9,24)}$, os quais avaliaram a sobrecarga e qualidade de vida de cuidadores de pessoas acometidas, sendo que em um dos estudos $^{(24)}$ os fatores explicaram aproximadamente $60 \%$ da variabilidade dos dados.

Em estudos anteriores que utilizaram esse mesmo questionário os cuidadores pesquisados obtiveram escores similares aos desse estudo ${ }^{(9,19)}$, principalmente no que se refere ao domínio emocional ${ }^{(9)}$.

Em consonância com os resultados encontrados nesse estudo, autores de uma pesquisa com cuidadores constataram que os mesmos obtiveram menores escores em aspectos emocionais comparado com o grupo controle ${ }^{(21)}$. Porém, na presente pesquisa, em relação aos aspectos físicos e globais ambos os grupos obtiveram escores semelhantes estatisticamente.
Os coeficientes de correlação (Spearman), obtidos através da relação linear entre as variáveis apontaram para uma forte ralação entre as próprias variáveis da componente para aspectos físicos (CAF) bem como entre as variáveis da componente para aspectos emocionais (CAE) e qualidade de vida em termos globais (QGV).

Em análise descritiva dos dados foi observado que cuidadores com idade inferior a 40 anos ou superior a 60 anos, possuem pior qualidade de vida em relação aos aspectos físicos e globais. Já em cuidadores com idades inferiores a 54 anos observou-se pior qualidade de vida em aspectos emocionais.

Em relação ao gênero dos cuidadores, foi observado que os homens obtiveram escores maiores do que as mulheres em todos os aspectos, enquanto que no grupo controle as mulheres apresentaram maior escore em aspectos físicos.

A utilização de testes não paramétricos (teste de MannWhitney) mostrou que estatisticamente não houve diferenças entre os escores dos índices CAF, CAE e QGV para os grupos controle e cuidadores, porém para o aspecto emocional tal evidência não é muito acentuada, sendo então a qualidade de vida entre os grupos semelhante em termos globais e físicos, mas na área emocional os cuidadores apresentam escores um pouco menores que o grupo controle. Estudos que utilizaram esse mesmo instrumento junto a cuidadores chegaram a resultados consonantes com os encontrados aqui ${ }^{(9,24)}$.

Pesquisas sobre cuidadores familiares que avaliaram aspectos sócio-demográficos como grau de parentesco, idade, estado geral dos acometidos, entre outras constataram que esses aspectos têm influência sobre a qualidade de vida dos sujeitos pesquisados ${ }^{(3,6,9,17,24)}$.

Em relação às covariáves para os cuidadores desse estudo, notou-se que para aspectos físicos, quanto maior a idade e o tempo em que o indivíduo exerce a função de cuidador, pior será sua qualidade de vida, sendo que cuidadores de cadeirantes têm pior qualidade de vida nesse domínio.

Nos aspectos emocionais, a qualidade de vida dos cuidadores é pior para pais e mães de afásicos. Quanto à qualidade de vida geral, ela é pior para aqueles que estão há mais tempo nessa função.

\section{CONCLUSÃO}

$\mathrm{Na}$ análise descritiva pôde-se perceber que os índices criados na análise fatorial (CAF, CAE e QVG) são bem representativos para os domínios dos cuidadores (e grupo controle) e refletem de maneira mais geral como está a qualidade de vida dos enfocados quanto aos aspectos físicos, emocionais e gerais.

Tanto em aspectos físicos quanto em aspectos gerais não foram encontradas diferenças significativas entre a qualidade de vida dos cuidadores e do grupo controle. Entretanto, nos aspectos emocionais o grupo de cuidadores apresentou menores escores, indicando assim pior qualidade de vida para esse grupo quanto a esse aspecto.

Em relação às variáveis demográficas consideradas no estudo verificou-se que a idade do cuidador, o grau de parentesco deste com o afásico e o tempo em que ele exerce a função de cuidador, são fatores que contribuem significativamente para alterações na qualidade de vida deles. 
Verificou-se, também, que a qualidade de vida desses cuidadores está comprometida em relação ao grupo controle, principalmente em aspectos emocionais.

$\mathrm{O}$ cuidador, tanto quanto o afásico, precisam se cuidar e precisam de cuidados, merecendo, então, atenção de profissionais da área da saúde, dentre eles o fonoaudiólogo, considerando-se que é fundamentalmente com o cuidador que se darão - ou não - os processos interativo-linguísticos do afásico, grandemente responsáveis pela sua recuperação lingüística. O cuidador, portanto, tem o potencial de reposicionar o sujeito afásico, tirando-o da condição de paciente e levando-o à condição de sujeito socialmente inserido.

A qualidade de vida de cuidadores de afásicos é um aspecto que merece ser estudado, tanto com vistas ao bem estar e à saúde do próprio cuidador quanto pelas conseqüências positivas ou negativas que o cuidar pode trazer para os afásicos,

Estudos posteriores devem ser realizados com maior variabilidade tanto do número de cuidadores quanto do seu perfil, enfocando-se, entre outros aspectos, diferentes níveis sócio-econômicos e diferentes quadros afásicos das pessoas sob cuidados.

Além disso, a grande variabilidade da faixa etária aqui enfocada também constitui aspecto a ser considerado em pesquisas posteriores uma vez que o estudo, em separado, de cuidadores de diferentes idades - adultos jovens, adultos, idosos de diferentes faixas etárias - deverá trazer dados específicos e de relevância para a maior compreensão da relação cuidador-afásico sob cuidados.

\section{AGRADECIMENTOS}

À Fundação de Amparo à Pesquisa do Estado de São Paulo (FAPESP) pelo apoio financeiro na forma de bolsa de iniciação científica (processo ${ }^{\circ} 07 / 53424-1$ ).

\begin{abstract}
Purpose: To evaluate the quality of life of caregivers of aphasic patients using the Medical Outcomes Study Short Form 36 (SF-36). Methods: Thirty caregivers of aphasic patients of both genders, with ages varying from 16 to 70 years, were analyzed. Thirty other individuals composed the control group, totalizing 60 subjects. All subjects were invited to answer to the questions of the SF-36. Moreover, the following variables were considered: gender, age, for how long have been occupying the position of caregiver, degree of kinship with the patient, and degree of the aphasic's motor impairment (gait). Univariate and descriptive regression analysis were carried out in order to test the associations among the variables. The scoring was statistically analyzed using the Mann-Whitney U test. Results: Variables such as age, time occupying the position of caregiver, degree of kinship with the patient, and degree of the aphasic's motor impairment (gait) influenced significantly the questions related to physical aspects. In the questions regarding emotional aspects only the variables age and kinship of the caregiver were significant. In the domain of global aspects, the only variable that influenced the final score was time acting as caretaker. In the questions related to physical and global aspects, the caregivers presented scores statistically similar to those of the control group, while in the questions regarding emotional aspects it was observed that they had lower scores than the control group. Conclusion: Regarding physical aspects and general conditions, caretakers and control group did not present considerable differences. However, regarding emotional aspects, caregivers had worse scores than the control group.
\end{abstract}

Keywords: Caregivers; Patient care; Aphasia; Health care; Quality of life; Stroke; Language; Questionnaires

\title{
REFERÊNCIAS
}

1. Coundry MIH. Diário de Narciso: discurso e afasia: análise discursiva de interlocuções com afásicos. 3a ed. São Paulo: Martins Fontes; 2001.

2. Michelini CRS, Caldana ML. Grupo de orientação fonoaudiológica aos familiares de lesionados cerebrais adultos. Rev CEFAC. 2005;7(2):13748.

3. Lavinsky AE, Vieira TT. Processo de cuidar de idosos com acidente vascular encefálico: sentimentos dos familiares envolvidos. Acta Sci Health Sci. 2004;26(1):41-5.

4. Schulz R, Martire LM. Family caregiving of persons with dementia: prevalence, health effects, and support strategies. Am J Geriatr Psychiatry. 2004;12(3):240-9. Review.

5. Guimarães Dos Santos CLN. Aspectos comportamentais em doenças crônicas: terapeuta, cuidador e paciente. In: Levy JA, Oliveira ASB, organizadores. Reabilitação em doenças neurológicas: guia terapêutico prático. São Paulo: Atheneu; 2003. p. 223-9.

6. Nakatani AYK, Souto CCS, Paulette LM, Melo TS, Souza MM. Perfil dos cuidadores informais de idosos com déficit de autocuidado atendidos pelo Programa de Saúde da Família. Rev Eletrônica Enferm. 2003;5(1):15-20.
7. Marques S, Rodrigues RAP, Kusumota L. Cerebrovascular accident in the aged: changes in family relations. Rev Latinoam Enferm. 2006;14(3):364-71.

8. Merino Carrera de Herrera SE. Calidad de vida de los cuidadores familiares que cuidan niños en situación de enfermedad crónica. Av Enferm. 2004;22(1):39-46.

9. Jönsson AC, Lindgren I, Hallström B, Norrving B, Lindgren A. Determinants of quality of life in stroke survivors and their informal caregivers. Stroke. 2005;36(4):803-8.

10. Mosca Filho M. Ética e terceira idade. In: Segre M. A questão ética e a saúde humana. São Paulo: Atheneu; 2006. p. 151-64.

11. Morato EM. Rotinas significativas e práticas discursivas: relato de experiência de um centro de convivência de afásicos. Distúrb Comun. 1999;10(2):157-65.

12. Morato EM, organizadora. Sobre as afasias e os afásicos: subsídios teóricos e práticos elaborados pelo Centro de Convivência de Afásicos (Universidade Estadual de Campinas). Campinas: Editora Unicamp; 2002.

13. Bookman A, Harrington M. Family caregivers: a shadow workforce 
in the geriatric health care system? J Health Polit Policy Law. 2007;32(6):1005-41.

14. Ferreira GC, Camargo EAA. Estudo de caso de um jovem afásico e sua qualidade de vida. Saúde Rev. 2005;7(15):33-8.

15. Bocchi SCM. Vivenciando a sobrecarga ao vir-a-ser um cuidador familiar de pessoa com acidente vascular cerebral (AVC): análise do conhecimento. Rev Latinoam Enferm. 2004;12 (1):115-21.

16. Bocchi SCM, Angelo M. Interação cuidador familiar-pessoa com AVC: autonomia compartilhada. Ciênc Saúde Coletiva. 2005;10(3):729-38.

17. Cattani RB, Girardon-Perlini NMO. Cuidar do idoso doente no domicílio na voz de cuidadores familiares. Rev Eletrônica Enferm. 2004;6(2):254-71.

18. Aranha LLM, Mirón Canelo JA, Alonso Sardón M, Del Pino Montes J, Sáenz González MC. Qualidade de vida relacionada à saúde em espanholas com osteoporose. Rev Saúde Pública $=$ J Public Health. 2006;40(2):298-303.

19. Martins T, Ribeiro JP, Garrett C. Estudo de validação do questionário de avaliação da sobrecarga para cuidadores informais. Psic Saúde \& Doenças. 2003;4(1):131-48.

20. Westphal AC, Alonso NB, Silva TI, Azevedo AM, Caboclo LOSF, Garzon E, et al. Comparação da qualidade de vida e sobrecarga dos cuidadores de pacientes com epilepsia por esclerose mesial temporal e epilepsia mioclônica juvenil. J Epilepsy Clin Neurophysiol. 2005;11(2):71-6.

21. Badia Llach X, Lara Surinach N, Roset Gamisans M. Calidad de vida, tiempo de dedicación y carga percibida por el cuidador principal informal del enfermo de Alzheimer. Atención Primaria. 2004;34(4):1707.
22. Hoe J, Katona C, Orrell M, Livingston G. Quality of life in dementia: care recipient and caregiver perceptions of quality of life in dementia: the LASER-AD study. Int J Geriatr Psychiatry. 2007;22(10):1031-6.

23. Sugiura K, Ito M, Mikami H. [Family caregiver burden caused by behavioral and psychological symptoms of dementia: measurement with a new original scale]. Nippon Ronen Igakkai Zasshi. 2007;44(6):717-25. Japanese.

24. Turró-Garriga O, Soler-Cors O, Garre-Olmo J, López-Pousa S, VilaltaFranch J, Monserrat-Vila S. Distribución factorial de la carga en cuidadores de pacientes con enfermedad de Alzheimer. Rev Neurol. 2008;46(10):582-8.

25. Belleza AMDO, Calegari VS, Raggio APR, Andrade GHM. Atuação fonoaudiológica em parceria com o "Programa Médico da Família" junto ao paciente com acidente vascular encefálico. Rev CEFAC. 2003;5(1): 31-9

26. Organização Mundial de Saúde. Divisão de Saúde Mental. Grupo WHOQOL. Versão em português dos instrumentos de avaliação de qualidade de vida (WHOQOL) 1998. Disponível em: http://www.ufrgs. br/psiq/whoqol.html; 2004.

27. Ferraz MBE, Ciconelli RM. Avaliação da qualidade de vida. In: Levy JA, Oliveira ASB, organizadores. Reabilitação em doenças neurológicas: guia terapêutico prático. São Paulo: Atheneu; 2003. p. 231-7.

28. Ciconelli RM, Ferraz MB, Santos W, Meinão I, Quaresma MR. Tradução para a língua portuguesa e validação do questionário genérico de avaliação de qualidade de vida SF-36 (Brasil SF-36). Rev Bras Reumatol. 1999;39(3):143-50.

29. Mendes PBMT. Quem é o cuidador. In: Dias ELF, Wanderley JS, Mendes RT. Orientações para cuidadores informais na assistência domiciliar. Campinas: Editora da Unicamp; 2002. p. 19-33. 